\title{
$=$

\section{Relación de la gestión del conocimiento y la competitividad en empresas de servicios}

\section{Relationship of Knowledge Management and competitiveness in service companies}

\author{
ESPARZA-GARCIA, Irma G. ${ }^{1}$ \\ SANCHEZ-GUERRERO, Marisol ${ }^{2}$ \\ CLARK-MENDIVIL, Yesenia ${ }^{3}$ \\ OLACHEA-PARRA, Luis F. ${ }^{4}$ \\ VELASCO-CEPEDA, Raquel $1 .{ }^{5}$
}

\begin{abstract}
Resumen
El artículo tiene como objetivo identificar la relación que existe entre la gestión del conocimiento y competitividad, en una muestra de empresas de servicios restaurantero y eventos en el municipio al Norte de México. Se utilizó correlación de Pearson para poner a prueba la hipótesis, y análisis factorial exploratorio para la confiabilidad de los constructos. Los resultados señalan, una relación positiva y significativa entre las variables de gestión del conocimiento y competitividad $(p<.001)$.

Palabras clave: gestión del conocimiento, competitividad, empresas de servicios.
\end{abstract}

\begin{abstract}
The article aims to identify the relationship between knowledge management and competitiveness, in a sample of restaurant service companies and events in the municipality of Northern Mexico. Pearson correlation was used to test the hypothesis, and exploratory factor analysis for the reliability of the constructs. The results indicate a positive and significant relationship between the variables of knowledge management and competitiveness ( $p<.001)$.

Keywords: knowledge management, competitiveness, service companies
\end{abstract}

\section{Introducción}

De forma gradual, la incertidumbre en los mercados y la globalización, además de los cambios sociales, económicos y tecnológicos, dan inicio a la era del conocimiento, esto se da finales de los años noventa, como respuesta de la creciente importancia para la efectividad organizacional (Bedoya, 2015). Este entorno ha provocado un escenario competitivo, en el que las empresas deben fortalecer sus ventajas competitivas a través

\footnotetext{
${ }^{1}$ Doctora en Planeación Estratégica para la Mejora del Desempeño por el Instituto Tecnológico de Sonora. Profesora Investigadora en el Departamento de Ciencias Administrativas del Instituto Tecnológico de Sonora. iesparza@itson.edu.mx

${ }^{2}$ Doctora en Gestión Organizacional. Profesora por asignatura-Investigador en Instituto Tecnológico Superior de Cajeme. msanchez@itesca.edu.mx.

${ }^{3}$ Doctora en Filosofía por la Universidad Autónoma de Nuevo León. Profesora Investigadora en el Departamento de Ciencias Administrativas del Instituto Tecnológico de Sonora. yessenia.clark@itson.edu.mx. Autor de correspondencia

${ }^{4}$ Doctor en Planeación Estratégica para la Mejora del Desempeño por el Instituto Tecnológico de Sonora. Profesor Investigador en Campus Guaymas del Instituto Tecnológico de Sonora. luis.olachea@itson.edu.mx

${ }^{5}$ Doctora en Planeación Estratégica para la Mejora del Desempeño por el Instituto Tecnológico de Sonora. Profesora Investigadora en el Departamento de Ciencias Administrativas del Instituto Tecnológico de Sonora. Raquel.velasco@itson.edu.mx
} 
de los activos de conocimiento (Marulanda, et al., (2016). El tema gestión del conocimiento se empleó por primera vez por el autor Karl M. Wiig en el año de 1986, definiéndolo como una construcción sistemática, renovadora y aplicación explícita y deliberada de conocimiento para incrementar la efectividad relacionada con el conocimiento de una empresa. Para los autores Voronov y Lavrinenko (2014), la gestión del conocimiento integra procesos organizacionales que explora una combinación sinérgica de la capacidad de procesamiento de datos e información de las tecnologías de la información y la capacidad creativa e innovadora de los seres humanos. Durante un tiempo considerable, las empresas contaban con la disposición de llevar al nivel consciente lo que sabían hacer, pero que hasta cierto punto no habían tenido tiempo de analizar; procuraron ir más allá de la comprensión de cómo poder hacer el mejor uso de los conocimientos que poseen, lo que las ha llevado a desarrollar procedimientos sistemáticos para gestionarlo (Cepeda, Martelo, Leal, Leal, 2017).

Transcendental en los últimos años se han producido cambios, donde el crecimiento de las empresas y por ende el de la economía, se han visto impulsadas por el conocimiento y las ideas, más, que por los recursos tradicionales (Del Moral, et asl.,2007). Derivado a que la sociedad se mueve hacia el conocimiento, donde los activos tangibles tradicionales están perdiendo valor a favor de los intangibles (Peña, 2001); Sánchez, et al.,(1999), es por ello que se puede considerar el conocimiento como el principal ingrediente intangible tanto en las empresas como en la economía en su conjunto (ocde, 1996).

Aunado a lo anterior los autores Hernández y Calderón (2011) expresan que la gestión del conocimiento se dirige hacia dos temas de estudio: la economía de la innovación y la economía de las competencias; por lo que se puede afirmar que el conocimiento es una forma de capacidad para actuar, procesar e interpretar información con la finalidad de generar más conocimiento que apoya en la toma de decisiones ante una problemática. Así mismo Nonaka y Takeuchi (1999) comentan que lo importante del conocimiento en las organizaciones depende de lo que se pueda hacer con él dentro de un ámbito de negocios, esto es, que el conocimiento por sí mismo no es relevante, en tanto no pueda ser utilizado para dar origen a acciones de creación de valor (Xiomara, 2009).

Es así como se puede observar que la intervención de la gestión del conocimiento dentro del ámbito empresarial, puede generar mayor ventaja competitiva en las mismas. Es por esto que el propósito de este estudio es revisar la intervención de la gestión del conocimiento como un factor de competitividad en empresas de servicios, y para lograrlo, se aplicó una metodología correlacional para posteriormente analizar su relación a nivel organizacional.

\section{Revisión literaria}

\subsection{Pymes de servicio}

Según la Secretaria de economía $([S E], 2018)$ las pymes son entidades independientes que tienen como objetivos la producción, trasformación y prestación de servicios para la satisfacción de necesidades de sus clientes. En México las pymes representan más del 3\% del total de empresas, y casi el 15\% del empleado del país, así mismo producen más del14\% del Producto Interno Bruto ([PIB]). En México, el $95.5 \%$ de las organizaciones manufactureras, de comercio y de servicios son microempresas, en tanto el $3.5 \%$ pequeñas, un $0.8 \%$ son medianas y sólo el 0.2 \% corresponde a las grandes empresas, de acuerdo con el Instituto Nacional de Estadística y Geografía ([INEGI], 2017).

Cabe destacar que el estado de Sonora cuenta con diversas actividades económicas, mostrando su riqueza en sus recursos naturales, además de su importante actividad industrial y principalmente en actividades del sector comercio y de servicios. La Situación actual de las mipymes en el estado de Sonora no es muy diferente al resto del país. Sin embargo, en el sector de giro servicio, con actividad económica preparación de alimentos y bebidas, 
localizadas en la zona urbana del sur de Sonora toma importancia según datos del INEGI (2017) ya que indican que contribuyen con el $17.56 \%$ del PIB estatal.

Este tipo de empresas de servicio tienen que encargarse de una serie de operaciones complejas en una cadena de valor global de proveedores y socios en constante evolución. Observando bajo la óptica y entorno internacional, se necesitan mejorar las relaciones con los socios y clientes, por ser mayormente competitivo, y por ende se suman mayores exigencias en sus operaciones, calidad y velocidad de producción y reducción de costos (Euroscrip, 2012). El servicio desde el punto de vista del turismo, debe ser estudiado en cuanto a las motivaciones de los usuarios, ya que eso permite comprender las expectativas, necesidades y los objetivos de los turistas, para poder así de forma fundamentada el diseñar estrategias de marketing, posicionamiento y competitividad (Carvache, Macas y Orden, 2018). Así mismo para Ortíz, et al.,(2019), el sector turismo en lo que respecta servicios, es un importante impulsador del desarrollo económico, debido a la cantidad de enlaces intersectoriales (operadores turísticos, agencias de viajes, empresas de transporte, hoteles, restaurantes).

Por tanto, este contexto hace enriquecedor el desarrollo de una investigación que se enfoque hacia los aspectos de correlación entre la gestión del conocimiento en pymes del sector servicios y por ende impacten a la competitividad.

\subsection{Teoría recursos y capacidades}

La teoría de recursos y capacidades consiste en la concepción de la empresa como un conjunto de recursos, donde se estudian los factores sobre los que se apoyan las ventajas competitivas, para poder determinar estrategias para una empresa. De acuerdo con Fernández y Suárez (1996), cada empresa es heterogénea ya que posee diferente dotación de recursos, producto de su historia y decisiones pasadas, sobre las que se puede sustentar una ventaja competitiva; también puede mantener esa heterogeneidad a lo largo del tiempo, es decir, la ventaja competitiva puede ser sostenible, lo que le proporcionara una renta a largo plazo. En estos últimos años, este enfoque está adquiriendo una relevancia y desarrollo en el ámbito de la estrategia empresarial, contrarrestando el excesivo peso que dentro del análisis estratégico han tenido los aspectos externos, concretados fundamentalmente en el modelo de fuerzas competitivas de Porter (1982). Para Garrido (2006), los recursos y capacidades de una organización pueden analizarse mediante la división de sus áreas, aspectos o funciones, de forma que se identifican diferencias sostenibles positivas o negativas las cuales deben ser modificadas o al menos esto se debe intentar.

Por otra parte, Zapata (2004), menciona que las empresas a través de su ciclo de vida van acumulando conocimiento que determina a través del mismo la capacidad de explotar y combinar todos sus recursos para el desarrollo de sus productos y servicios. Es decir, la teoría considera que los recursos son valiosos cuando permiten a la organización mejorar la efectividad, aprovechar las oportunidades y neutralizar las amenazas. El conocimiento desde el punto de vista como un recurso, se centra en las capacidades dinámicas, entendidas como la habilidad de la organización para integrar, construir y reconfigurar su dotación de recursos y capacidades para responder rápidamente a los cambios del entorno (López \& Sabater, 2002). Es así como el conocimiento puede ser considerado como uno delos recursos estratégicos más importantes.

\subsection{Gestión del conocimiento}

El conocimiento es considerado como una mezcla de experiencias estructuradas, de valores y contextos que proporcionan un marco de evaluación e incorporación de nueva información Davenport y Prusak (2000). Además, ya anteriormente el conocimiento fue nombrado por Nonaka y Takeuchi (1995), como ese proceso dinámico que acredita la búsqueda personal de la verdad y es centrada en niveles de entidades creadoras. Así pues, toda vez identificado el conocimiento que es sistematizado y compartido entre todos los miembros de la 
organización se logra la gestión del conocimiento, que se transforma en una fuente de creación de valor para toda organización.

Con el paso del tiempo se han incorporado diversas definiciones en relación a la gestión del conocimiento, por ejemplo Castillo y D’Vicente (2009), en su investigación la definen como un proceso donde se planifica, organiza, integra y controla el acceso y uso oportuno de información que es convertida en un conocimiento colectivo y de formación, donde el primero se da a través de un conocimiento tácito (consta comúnmente de experiencias, educación, hábitos y aspectos culturales) y el segundo conocimiento explícito (hace referencia a aquel que ha sido o puede ser articulado, codificado y almacenado en algún tipo de medio, que puede ser transmitido inmediatamente a otros); provocando juntos el incremento de la productividad e innovaciones en los productos y servicios de la organización.

Por su parte Contreras y Díaz (2014), conceptualizaron la gestión del conocimiento como la creación, transferencia, evaluación, disponibilidad y puesta en práctica del conocimiento. Observándola como un enfoque orientado a localizar y aplicar las mejores prácticas procesos para lograr ventajas competitivas sostenibles en la organización.

También Meihami y Meihami (2014), definen a la gestión del conocimiento como una forma de crear valor a través del estímulo de los conocimientos, la creatividad e innovación, el uso de herramientas y técnicas específicas y experiencias dentro y fuera de la organización, de tal manera que el conocimiento se encuentra disponible cuando la organización necesite tomar decisiones que transcienden en el proceso.

Aunado a lo antes señalado Tinoco, Salas y Tinoco (2014), vinculan a la gestión del conocimiento como una necesidad estratégica empresarial, ya que consideran está representada tanto por el saber individual como el organizativo, siendo pues un activo de valor al ser aplicado en el proceso general de la empresa.

Posteriormente el autor Sukmawati (2015), afirma que la gestión del conocimiento se utiliza como una actividad para alcanzar el objetivo de la organización, acondicionándola para superar los desafíos del entorno interno y externo en el que se desenvuelve para mantener la competitividad dentro del mercado. Además, Rodríguez, Nuñez y Esparza (2018), mencionan que la gestión del conocimiento es un elemento importante para generar ventajas sostenidas.

Así mismo a través de su estudio Sánchez (2016), pudo concluir que la gestión del conocimiento se encuentra integrada de un proceso dinámico en el que se obtiene información y al ser transformada se adquiere conocimiento; con este conocimiento se pueden desarrollar estrategias que contribuyen al desarrollo y mejoramiento de una organización. Abriéndose con ello hacia dos campos de estudio como la economía de la innovación y la economía de las competencias.

En general, la literatura provee que la gestión del conocimiento hace referencia a la necesidad que posee una empresa en implementar un sistema de información que sirve como plataforma de apoyo de los procesos organizativos de creación, almacenamiento, transferencia y aplicación de conocimiento. También se observa una asociación con el proceso de aprendizaje y la toma de decisiones donde el objetivo fundamental es lograr la sinergia entre las capacidades y la creatividad de los miembros de la organización, contribuyendo a la competitividad empresarial.

\subsection{Competitividad}

La competitividad es un elemento primordial para que las pymes mantengan ventajas comparativas que le permiten disfrutar y sostener una posición destacada en el entorno socioeconómico en que actúan. Así pues, Pérez (2008), manifiesta que se entiende por ventaja comparativa aquellas habilidades, recursos, conocimientos, 
atributos, etc., de que dispone una empresa, de la que carecen sus competidores y que hace posible la obtención de unos rendimientos superiores a estos.

El autor Porter (2008), en su artículo "Cómo las fuerzas competitivas le dan forma a la estrategia" dice que la ventaja tiene que ver en lo fundamental con el valor que una empresa es capaz de crear para sus compradores. El concepto de competitividad es relativo, y que muestra una posición comparativa de las empresas al utilizar la misma medida de referencia. Se puede decir que es un concepto aun en desarrollo, y se utiliza según la dimensión a la que pertenezcan los sistemas organizativos, se utilizarán indicadores distintos para medirla.

Por otro lado y observando a la competitividad como las competencias profesionales los autores Vázquez y Rojas (2012), mencionan que está relacionada al conjunto de conocimientos, destrezas, comportamientos y motivaciones que tienen una relación con el desempeño sobresaliente de las personas en una actividad competitiva; concluyendo con ello, que la competitividad es la capacidad de las organizaciones para diseñar, desarrollar, producir y colocar sus productos en el mercado internacional en medio de la competencia con empresas de otros países.

Por su parte Licona (2013), hace mención en que la competitividad bajo otro enfoque el cual consiste en la capacidad que tiene una empresa o bien un país para mantener e incrementar su contribución en las organizaciones mundiales, manifestando con ello una mayor competitividad entre las asociaciones y países por lograr más lugares en la economía universal.

Con lo antes descrito es evidente el no contar con un consenso universal a cerca del concepto competitividad, según Larios (2016), debe ser entendida desde tres ópticas, una empresarial a través de un enfoque micro, otra desde lo económico con un enfoque meso y la tercera de forma global a través de un enfoque macro.

Finalmente, se observa una interiorización ente la gestión del conocimiento y la competitividad ya que juntas facilitan el uso de herramientas de ayuda a la comunicación, análisis de información, aprendizaje, innovación, creatividad y desarrollo organizacional, ya que el reto en esta era es generar nuevos conocimientos a partir de los ya formalizados, es decir, generar conocimientos tácitos con base en los explícitos, contribuyendo al buen desempeño organizacional, por lo anterior y para confirmar tal afirmación se han planteado una hipótesis de investigación:

H1. La gestión del conocimiento se asocia de manera positiva y significativa con la competitividad.

\section{Metodología}

La presente investigación es de tipo cuantitativo, de alcance descriptivo y correlacional, no experimental de corte transversal. El instrumento de estudio fue basado en la Red Centro occidente de competitividad. Cabe señalar que el instrumento se integró por una escala tipo Likert con cinco opciones de respuesta, las cuales variaban desde 1 (Muy en descuerdo) hasta 5 (Muy de acuerdo), en donde una mayor puntuación indica una mayor concordancia o valoración atribuida a cada responsabilidad en particular. La operacionalización de variables se observa en la tabla 1, donde competitividad se conforma por desempeño financiero, costos de compra y tecnología. Mientras que gestión del conocimiento por capacitación, creatividad e innovación, conocimiento y motivación. 
Tabla 1

Operacionalización de variables

\begin{tabular}{llc}
\hline \multicolumn{1}{c}{ Variable } & \multicolumn{1}{c}{ Dimensiones } & Reactivos \\
\hline \multirow{3}{*}{$\begin{array}{l}\text { Gestión del } \\
\text { conocimiento }\end{array}$} & Capacitación & 5 \\
& Creatividad e innovación & 13 \\
& Conocimiento & 5 \\
& Motivación & 4 \\
& Desempeño financiero & 6 \\
Competitividad & Costos de compra & 6 \\
& Tecnología & 6 \\
\hline
\end{tabular}

Para su procesamiento se empleó SPSS (Statical Package for the Social Sciences). La muestra se constituyó por 60 empresas de tamaño pymes de servicio restaurantero y eventos del municipio de Cajeme, Sonora México (Tabla 2).

Para la validez del instrumento de medición, se contempló la técnica de análisis factorial exploratorio, por medio de análisis de componentes principales con rotación Varimax para medir la validez de constructo, la cual se refiere al grado en que un instrumento realmente mide la variable que pretende medir. Los resultados de la prueba indicaron un índice Kaiser-Meyer-Oklin de 0.820, una prueba de esfericidad de Bartlett $\left(X^{2}=1039.258, \mathrm{gl}\right.$ $=153, \mathrm{p}<.000)$ para el constructo de Competitividad y un índice Kaiser-Meyer-Oklin de 0.812, prueba de esfericidad de Bartlett $\left(X^{2}=1590.863, \mathrm{gl}=351, \mathrm{p}<.000\right)$ para el constructo de gestión del conocimiento y cargas factoriales mayores a 0.539 en todas las preguntas (Hair, Anderson, Tatham, y Black 2004).

Tabla 2

Caracterización de la muestra

\begin{tabular}{lc}
\hline \multicolumn{1}{c}{ Categoría } & $\%$ \\
\hline $\begin{array}{l}\text { Sector servicio restaurante } \\
\text { Sector servicio eventos } \\
\quad \text { El control de la empresa es familiar }\end{array}$ & 50 \\
Sí & 63 \\
No & 37 \\
Puestos directivos ocupados por miembros de la familia & \\
Sí & 60 \\
No & Género del puesto directivo \\
Masculino & 40 \\
Femenino & 75 \\
\hline
\end{tabular}

Fuente: Elaboración propia

La formación académica de los participantes resulto con mayor presencia en nivel superior con un $70 \%$ seguido del medio básico con un $20 \%$ y básico con el $10 \%$. Cabe mencionar que en todo momento se cuidó que el tamaño de muestra fuera el adecuado para los análisis estadísticos correspondientes (Levin \& Rubin, 2004; Pérez, 2004).

\section{Resultados}

Con el propósito de poner a prueba la hipótesis de investigación se empleó la técnica de correlación de Pearson para medir la asociación entre las variables. 
Se verificó la confiabilidad, la cual se refiere al grado de consistencia de un instrumento, cuyos valores alpha de cronbach deben ser $\geq 0.7$ (Montoya, 2007) (Tabla 3).

Tabla 3

Niveles de confiabilidad

\begin{tabular}{llc}
\hline \multicolumn{1}{c}{ Variable } & \multicolumn{1}{c}{ Factor } & Alpha de Cronbach \\
\hline Gestión del & $\begin{array}{l}\text { Capacitación } \\
\text { Conocimiento }\end{array}$ & .886 \\
& $\begin{array}{l}\text { Conocimiento } \\
\text { Motivación }\end{array}$ & \\
Competitividad & $\begin{array}{l}\text { Desempeño financiero } \\
\text { Costos de compra }\end{array}$ & .789 \\
& Tecnología & \\
\hline
\end{tabular}

Fuente: Elaboración propia

Los resultados se confirman por medio de correlación de Pearson, donde se observa que la gestión del conocimiento y la competitividad se correlacionaron de manera positiva y significativa a un nivel de confianza del 99\% (Tabla 4); lo cual significa que, a mayor gestión del conocimiento referido a capacitación, creatividad e innovación, conocimiento y motivación mayor será la competitividad de las pymes restauranteras y de eventos en su desempeño financiero, costos de compra y tecnología.

Tabla 4

Correlación de las

variables de estudio

\begin{tabular}{llcc}
\hline & & $\begin{array}{c}\text { Gestión del } \\
\text { conocimiento }\end{array}$ & Competitividad \\
\hline Gestión del & Correlación de Pearson & 1 & $.632 * *$ \\
conocimiento & Sig. (bilateral) & 60 & .000 \\
& $\mathrm{~N}$ & $.632 * *$ & 60 \\
Competitividad & Correlación de Pearson & .000 & 1 \\
& Sig. (bilateral) & 60 & 60 \\
\hline
\end{tabular}

Fuente: Elaboración propia

\footnotetext{
* La correlación es significativa al nivel 0.01 (bilateral).

** La correlación es significante al nivel 0.05 (bilateral).

*** La correlación es significativa al nivel 0.1 (bilateral).
}

Dicha evidencia empírica soporta la hipótesis de que la gestión del conocimiento se relaciona para que una empresa llegue a ser competitiva. Esto es a mayor capacitación, innovación, conocimiento y motivación exista en la organización, mayor será el mejoramiento del desempeño financiero los costos de compra y la tecnología.

Los presentes resultados pueden ser empleados como evidencia empírica para soportar la relación entre gestión del conocimiento y competitividad, tal como se propone. Sin olvidar, que hay escases de resultados empíricos en pymes, por lo cual es una necesidad realizar más estudios sobre el tema.

\section{Conclusiones}

El objetivo de la presente investigación fue analizar la relación de la gestión del conocimiento y la competitividad. Se pudo probar la hipótesis planteada. A través de los hallazgos ya que se pudieron encontrar elementos que sostienen a la gestión del conocimiento a través de los factores capacitación, creatividad e innovación, conocimiento y motivación, también pueden ayudar al desarrollo de la competividad mostrado a través del desempeño financiero, los costos de compra y la tecnología. 
El análisis de la competitividad empresarial en el presente estudio comprende tres tipos principales de elementos: desempeño financiero, costos de compras y tecnología. Todos ellos, de manera conjunta, definen la posición competitiva de las empresas y proveen los elementos necesarios para realizar análisis orientados a mejorar el desempeño de las empresas de este giro.

Cabe señalar que este estudio es un primer intento empírico desarrollado en pymes de servicio restaurantero y de eventos para mostrar la asociación entre las variables de estudio, los cuales son recursos valiosos para las empresas, a través del cobijo de la teoría de recursos y capacidades. Es preciso mencionar que no se localizó evidencia sobre algún trabajo teórico o empírico semejante.

Se sugiere realizar un nuevo estudio con una muestra más grande. Asimismo, se recomienda la realización de un estudio longitudinal para medir niveles de causalidad. Sería conveniente replicar la investigación en otro sector donde se disponga de una población más abundante.

Asimismo, los resultados son un punto de partida para otras investigaciones que busquen asociar a gestión del conocimiento y competitividad medida con otras variables o bien factores, la cuales han cobrado mucha relevancia dentro de la actual era del conocimiento.

\section{Referencias bibliográficas}

Bedoya Dorado, C. (2015). Gestión del conocimiento en el tercer sector: de la competitividad a la eficiencia organizacional. Entramado, 11(2), 94-111. Recuperado de: http://revistasojs.unilibrecali.edu.co/index.php/entramado/article/viewFile/619/786

Castillo, M., y D’Vicente, Y. (2009). Gestión del conocimiento y competitividad en el área de investigación y producción de los colegios e institutos universitarios privados de Maracaibo, Venezuela. Clío América, 3(6), 165-182.Recuperado de file://C:/Users/Usuario/Downloads/389-1-918-1-10-20140131.pdf

Carvache, M., Carvache, W., Macas, C., y Orden, M. (2018). Motivaciones, Valoración y Satisfacción del Turista en un destino de Sol y Playa de Ecuador. Revista Espacios, 39(13), 4-20.

Cepeda-Carrión, I., Martelo-Landróguez, S., Leal Rodríguez, A. L., y Leal-Millán, A. (2017). Critical processes of knowledge management: An approach toward the creation of customer value. European Research on Management and Business Economics, 23(1), 1-7. Recuperado de: https://ac.elscdn.com/S2444883416300092/1-s2.0-S2444883416300092main.pdf?tid=312c1111-f92c-4b53adcd79a2bdbc7310\&acdnat=1531097227_1eb91c97209e6699c5485bfda44687c0

Contreras-Medina, D. I., y Díaz-Nieto, E. S. (2014). La gestión del conocimiento factor clave de competitividad. Un estudio de los modelos y paradigmas. Contribuciones a la Economía.Recuperado de: http://www.eumed.net/ce/2014/2/conocimiento-competitividad.html

Davenport, T. H. and Prusak, L. Working knowledge: How organizations manage what they know. MA: Harvard Business School Press, Boston, 2000.

Del Moral, A. D., Pazos, J., Rodríguez, E., Rodríguez, A., \& Suárez, S. (2007). Gestión del conocimiento. España: Thompson Editores.

Euroscrip, (2012). Industria manufacturera. Euroscript International. Recuperado de: http://www.euroscript.com/luxembourg/es/35.html

Fernández, Z. y Suárez, I. (1996). La Estrategia de la Empresa desde una Perspectiva basada en los Recursos. Revista Europea de Dirección y Economía de la Empresa, 5, 73-92 
Garrido B., S. (2006). Dirección estratégica. 2ª. ed. España: McGraw Hill Hernández, F. O., \& Calderón, J. M. (2011). Las Instituciones de Educación Superior organizaciones generadoras del trabajador del conocimiento. (Spanish) Administración y Organizaciones, 14(26).

Cair F., AndersonR., y BlackW. (2004). Análisis multivariante. 5ạ. Ed. España: Prentice Hall

INEGI (2017). Principales resultados del Censo de Población y Vivienda 2017. Recuperado de: http://www.inegi.gob.mx/prod_serv/contenidos/espanol/bvinegi/productos/censos/poblacion/2017/prin ci_result/cpv2017_principales_resultadosl.

Larios Gómez, E. (2016). La gestión de la competitividad en la mi pyme mexicana: diagnóstico empírico desde la gestión del conocimiento. Revista de Administração da Unimep, 14(2), 177-209. Recuperado de: http://www.redalyc.org/pdf/2737/273746863008.pdf

Levin, R. I., \& Rubin, D. S. (2004). Estadística para administración y economía (7.a ed.). México: Pearson Educación de México, S. A. de C. V.

Licona Michel, Á. \&. (2013). Pilares de la competitividad, educación superior, nuevas tecnologías y empleo en Corea del Sur y México. Análisis Económico, 28(69), 79-108.

López Y, J. A. y Sabater S., R. La Teoría de los Recursos y Capacidades de la Empresa. Una Revisión. 2002. Departamento de organización de Empresas. Universidad Murcia, pp. 1-30. En línea. Internet. 23 febrero 2007. Recuperado de: http://www.um.es/fee/documentos/dt2-00.pdf

Marulanda, C., López, M., y López, F. (2016). La cultura organizacional y las competencias para la gestión del conocimiento en las pequeñas y medianas empresas (pymes) de Colombia. Información tecnológica, 27(6), 03-10.

Meihami, B., y Meihami, H. (2014). Knowledge Management a way to gain a competitive advantage in firms (evidence of manufacturing companies). International Letters of Social and Humanistic Sciences, 14(3), 8091. Recuperado de: file:///C:/Users/Usuario/Downloads/article_176912.pdf

Montoya S. O. (2007). Evaluación de confiabilidad y validez de una versión en español del cuestionario breve impacto del peso en la calidad de vida en pacientes de la clínica Reina Sofía en Bogotá a os 20062008.Médica. Sanitas. 12(3), 18-35.

Nonaka, I., and Takeuchi, H. (1995). The knowledge-creating company: How Japanese companies create the dynamics of innovation .London Oxford University Press.

Ocde (1996). The Knowledge-Based Economy, Mimeo, París, ocde, Mimeo: 1-46.

Peña, P. (2001) .To know or no to be. Conocimiento, el oro gris de las organizaciones. Dintel, Madrid.

Ortíz, J.S.; Cancino, S. E. y Cancino, G.O. (2019). El aporte del turismo al desarrollo económico del Municipio de Pamplona, región nororiental de Colombia. Revista Espacios, 40(01), 15-24.

Pérez, C. (2004). Técnicas de análisis multivariante de datos: aplicaciones con SPSS. Madrid, España: Pearson Educación, S. A.

Pérez, A. y Pérez, O. (2008). Competitividad y acumulación de capacidades tecnológicas en la industria manufacturera mexicana. Universidad Autónoma de Zacatecas. Recuperado de: http://redalyc.uaemex.mx/src/inicio/ArtPdfRed.jsp?iCve=60111154005. 
Porter, M. (1982). Estrategia competitiva: Estrategias para el análisis de los sectores industriales y de la competencia. Madrid: CEC SA.

Porter, M.E. (2008). The five competitive forces that shape strategy. Harvard Business Review, 86(1), 78-93.

Rodríguez, M.J., Nuñez, M.A., y Esparza, I.G. (2018). Prueba empírica del modelo de Gestión del Conocimiento de Nonaka y Takeuchi en el contexto universitario. Revista Espacios, 39(49), 36-47.

Sánchez, M; Chaminade, C; Escobar, C. (1999). En busca de una teoría sobre medición y gestión de los intangibles en la empresa: Una aproximación metodológica. Ekonomiaz, 45, 188-213.

Sánchez, J. (2016). La gestión del conocimiento y la competitividad en las pymes. Guadalajara, México: Editorial Universidad de Guadalajara.

Secretaría de Economía (SE). Programa de Desarrollo Empresarial Informe 2017-2018. Recuperado de: de https://www.gob.mx/cms/uploads/attachment/file/383749/09_Metropolitana_Informe_Actividades_201 7-2018.pdf

Sukmawati, S. (2015). A knowledge management strategy to achieve organization competitiveness. International Journal of Scientific \& Technology Research, 4(8), 334-339.

Tinoco Gómez, O. R., Salas Bacalla, J. A., y Tinoco Ángeles, F. E. (2014). Gestión del conocimiento y su incidencia en la competitividad empresarial de las mypes del sector textil y de confecciones en Lima Metropolitana. Theorēma - UNMSM, 1(1),161-170. Recuperado de: file://C:/Users/Usuario/Downloads/11949-41663-1PB.pdf

Vázquez M. L y Rojas M. H. Las competencias profesionales en la gestión de la in-formación y del conocimiento en salud. MEDICIE-GO [Internet]. 2012 [citado 23 Oct 2014];18(3): [aprox. 7 p.]. Recuperado de: http://bvs.sld.cu/revistas/mciego/vol18_02_12/articulos/t-3.html

Voronov, V., y Lavrinenko, O. (2014). Knowledge management and the incresase of SME competitiveness: a case study. Baltic Region, 3(21), 22-32. Recuperado de: https://cyberleninka.ru/article/n/knowledgemanagement-and-the-incresase-ofsme-competitiveness-a-case-study

Xiomara, P. (2009). La gestión del conocimiento y las Tics en el siglo xxi. Conhisremi. Revista Universitaria de Investigación y Diálogo Académico, 5(1).

Esta obra está bajo una Licencia Creative Commons Attribución-NoCommercial 4.0 International

$(\mathrm{Cc}) \mathrm{BY}$-NC 